\title{
The Pseudomonas syringae effector AvrPtoB targets abscisic acid signaling pathway to promote its virulence in Arabidopsis
}

Yanzhi Liu ${ }^{1,2}$, Md. Rubel Mahmud ${ }^{1,4}$, Ning $\mathrm{Xu}^{3^{*}}$ and Jun Liu ${ }^{3^{*}}$

\begin{abstract}
Phytohormones play an essential role in plant immune responses. Many phytopathogens secret effector proteins to promote infection and plant hormone signaling pathways are considered to be the potential targets of effectors. Here we found that abscisic acid (ABA) signaling was activated rapidly upon infection with Pseudomonas syringae pv. tomato (Pst). Pst secretes the effector AvrPtoB to target ABA 8'-hydroxylase CYP707As for degradation in Arabidopsis thaliana. CYP707As hydroxylate ABA to an inactive form. The degradation of CYP707As resulted in ABA accumulation and compromised plant immune responses. Our study demonstrated that Pst could hijack the key components of Arabidopsis ABA signaling pathway to cause disease.
\end{abstract}

Keywords: Pseudomonas syringae, ABA, AvrPtoB, CYP707A

\section{Background}

Due to immovable feature, plants are constantly challenged by abiotic and biotic stresses, such as drought, high salinity and pathogens. Many phytohormones have been demonstrated to play essential roles in plant immune response. Of the investigated hormones, salicylic acid (SA), jasmonic acid (JA) and ethylene are the most important ones in plant basal defenses ( $\mathrm{Li}$ et al. 2019; Ding and Ding 2020). The phytohormone ABA (abscisic acid) is known to regulate plant responses to abiotic stresses, but its role in biotic stress responses remains inconclusive and controversial (Cutler et al. 2010; Cao et al. 2011; Chen et al. 2020).

In response to stress, ABA binds to its receptors PYRABACTIN RESISTANCE1(PYR1)/PYR1-LIKE (PYL) and mediates the binding to clade A protein phosphatase2Cs (PP2Cs), leading to suppression of phosphatase activities.

\footnotetext{
*Correspondence: ningxu@cau.edu.cn; Junliu@im.ac.cn

${ }^{3}$ Department of Plant Pathology, MOA Key Lab of Pest Monitoring

and Green Management, College of Plant Protection, China Agricultural University, Beijing 100193, China

Full list of author information is available at the end of the article
}

This results in the immediate release of sucrose nonfermenting-1 (SNF1)-related protein kinases (SnRK2s), and induces stomatal closure as well as downstream gene expression via the phosphorylation of S-type anion channels and some transcription factors, such as ABI5 (a basic leucine zipper transcription factor) and HAT1 (an HD-ZIP II transcription factor) (Meyer et al. 1994; Ma et al. 2009; Umezawa et al. 2009; Brandt et al. 2012; Dai et al. 2013). Endogenous ABA levels are regulated by both biosynthesis and catabolism (Nambara and Marion-Poll 2005). The Arabidopsis cytochrome P450 (CYP) super-family genes CYP707A encode ABA 8'-hydroxylases. These enzymes catalyze the first committed step in $\mathrm{ABA}$ catabolic pathway, resulting in the production of 8'-hydroxy ABA. 8'-hydroxy ABA is then isomerized spontaneously to phaseic acid (PA), leading to the significant reduction in biological activity of ABA (Kushiro et al. 2004; Saito et al. 2004). In Arabidopsis, there are four $C Y P 707 A$ homolog genes; of which the expression of CYP707A1 is dramatically induced by exogenous ABA application (Okamoto et al. 2006). Arabidopsis cyp707a single and double mutants can accumulate high levels of original author(s) and the source, provide a link to the Creative Commons licence, and indicate if changes were made. The images or other third party material in this article are included in the article's Creative Commons licence, unless indicated otherwise in a credit line to the material. If material is not included in the article's Creative Commons licence and your intended use is not permitted by statutory regulation or exceeds the permitted use, you will need to obtain permission directly from the copyright holder. To view a copy of this licence, visit http://creativecommons.org/licenses/by/4.0/. 
ABA in seeds, whereas CYP707A overexpression lines display lower ABA levels (Kushiro et al. 2004; Okamoto et al. 2006), suggesting the key roles of $C Y P 707 A$ in $A B A$ accumulation.

ABA not only regulates stomatal closure, leaf abscission, seed germination and dormancy, but also regulates plant responses to a wide range of biotic stresses. However, the effect of ABA signaling on basal defenses depends on the type of pathogens. ABA-deficient mutants aba1-6, abi1-1 and abi2-1 exhibit enhanced susceptibility to the soil-borne bacterium Ralstonia solanacearum, but they are resistant to infection of the necrotrophic fungus Plectosphaerella cucumerina (Hernández-Blanco et al. 2007). The ABA biosynthesis mutants aba2-12, aao3-2 and ABA-insensitive mutant abi4-1 show enhanced susceptibility to oomycete pathogen Pythium irregular, necrotrophic pathogen Alternaria brassicicola, but exhibit strong resistance to necrotrophs Botrytis cinerea (Adie et al. 2007); while ABA biosynthesis mutant aba3-1 is susceptible to biotrophic oomycete pathogen, Hyaloperonospora arabidopsis (Fan et al. 2009). By contrast, the ABA biosynthesis mutants $a b a 2-1$ and $a b a 3-1$ display enhanced resistance to the biotrophic powdery mildew fungus Golovinomyces cichoracearum (Xiao et al. 2017).

During Pseudomonas syringae infection, ABA plays a positive role in pre-invasive stomatal immunity by inducing stomatal closure to prevent pathogen entry; however, it plays a negative role in post-invasive immunity (Cao et al. 2011). aba3-1, aba2-3 and pyr1-2 are more resistant to $P$. syringae by syringe infiltration (García-Andrade et al. 2020). Application of exogenous ABA enhances plant susceptibility to Pst and Pst $\mathrm{hrpA}^{-}$, a type III protein secretion system (T3SS)-defective mutant (de Torres-Zabala et al. 2007; Fan et al. 2009; Tan et al. 2019; García-Andrade et al. 2020). Notably, Pst infection has been reported to induce the accumulation of endogenous $\mathrm{ABA}$, which is likely one of the reasons that this pathogen causes disease in Arabidopsis plants (de Torres-Zabala et al. 2007; Gao et al. 2016).

Many phytopathogens deploy effector proteins to subvert host immune response or target susceptible genes to promote infection. Pst, for instance, can deliver a set of effector proteins to host cells, which dramatically suppress host immune responses. Introducing Pst effector HopAM1 to Arabidopsis markedly increases water availability and colonization ability of the pathogen. HopAM1 also suppresses host basal defense and improves the sensitivity to ABA in plants (Goel et al. 2008). Likewise, the effector protein AvrPtoB has E3 ligase activity and can target host receptor-like kinases (RLKs) such as FLS2, CERK1 and LecRK-IX.2 for degradation, which subsequently suppresses the immune responses mediated by these receptors (Janjusevic et al. 2006; Göhre et al. 2008; Gimenez-Ibanez et al. 2009; Xu et al. 2020). The Arabidopsis genome harbors 23 EXO70 protein family members, some of which are involved in plant immunity. AvrPtoB can ubiquitinate and mediate the degradation of EXO70B1 to overcome EXO70B1-mediated resistance (Wang et al. 2019). Conditional expression of AvrPtoB in Arabidopsis results in a significant increase in ABA level and an enhanced susceptibility to Pst $\mathrm{hrpA}^{-}$(de TorresZabala et al. 2007).

We previously demonstrated that AvrPtoB targets LecRK-IX.2 for degradation, leading to immune suppression in host plants. AvrPtoB can also mediate the degradation of NON-EXPRESSER OF PR1 GENES1 (NPR1) to interfere with SA signaling and subvert plant innate immunity (Chen et al. 2017). However, how AvrPtoB manipulates host's ABA signaling pathway is unclear. In this study, we reveal that AvrPtoB targets ABA 8'-hydroxylase CYP707As for degradation, which subsequently facilitates ABA accumulation and promotes Pst infection.

\section{Results}

cyp707a mutants demonstrate compromised PTI responses Recognition of pathogen- or microbe-associated molecular patterns (PAMPs or MAMPs) is essential for plants to distinguish self- and nonself-components (Zipfel 2014). To explore this process, we screened a stock of Arabidopsis mutants by treating with flg22, a peptide of bacterial flagellin that can trigger strong PTI response (Gómez-Gómez et al. 1999). Two mutants, cyp707a1 (cyp707a1-1, SALK_069127) and cyp707a2 (cyp707a2-2, SALK_083966) showed a reduction in both flg22-induced ROS production and the expression of PTI responsive gene FRK1 (Fig. 1a and Additional file 1: Fig. S1a) (Asai et al. 2002). These two mutants also showed a reduced ROS burst by another immune elicitor elf18 that is derived from translation elongation factor $\mathrm{Tu}$ of bacterial pathogens (Fig. 1b). Pathogen-induced callose deposition has been reported to function as a chemical and physical defense mechanism for host to avoid pathogen attack (Kunze et al. 2004). We then examined the callose deposition in plant leaves treated with flg22. The result showed that flg22-induced callose deposition was significantly suppressed in both cyp707a1 and cyp707a2 mutants when compared to Col-0 (Fig. 1c, d). The expression of GSL6, a gene encoding GLUCAN SYNTHASELIKE (GSL) callose synthases, was also remarkably down-regulated in the two mutants (Additional file 1: Fig. S1b). MAPK activation is one of the early events that can be triggered by various PAMPs molecules. The flg22induced MAPK activation displayed slight reduction in cyp707a2 mutant compared with that in Col-0 (Additional file 1: Fig. S1c). The callose deposition also reduced 
a



C
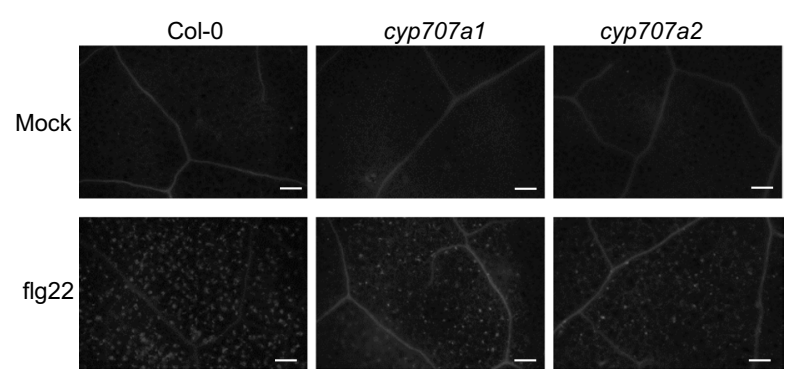

b
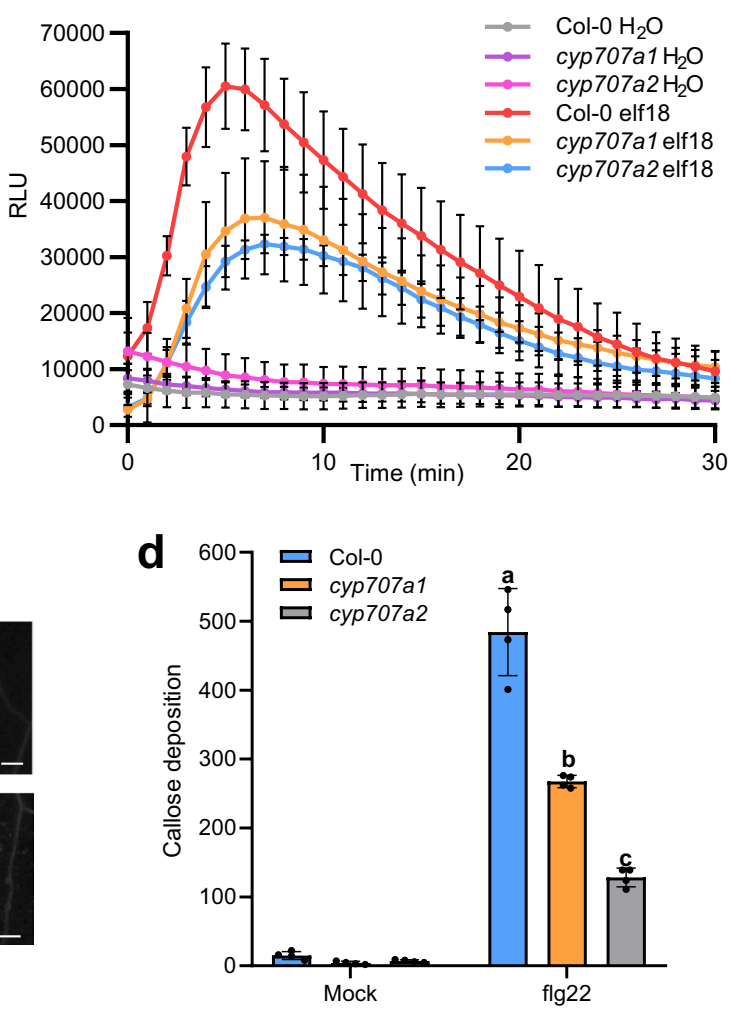

Fig. 1 The cyp707a mutants display reduced PTI response. a and b flg22- and elf18-induced ROS burst is reduced in cyp707a mutants. Leaf discs from three-week-old Arabidopsis plants were treated with $100 \mathrm{nM}$ flg22 or $100 \mathrm{nM}$ elf18, and the ROS burst was recorded. $\mathrm{H}_{2} \mathrm{O}$ served as a control. Error bars represent means $\pm S D$ ( $n=3$ biological replicates). RLU, Relative light units. $\mathbf{c}$ and $\mathbf{d}$ cyp707a mutants display decreased callose deposition in response to flg22 treatment. Four-week-old Col-0 and cyp707a mutants were syringe infiltrated with $\mathrm{Mock}(10 \mathrm{mM} \mathrm{MgCl}$ ) or $100 \mathrm{nM}$ flg22. The calloses were stained with aniline blue (c) and counted under microscope (d) at 12 hpi (hours post-inoculation). Scale bar $=100 \mu \mathrm{m}$. Error bars represent means \pm SD (Two-way ANOVA, $P<0.001, n=4)$

in cyp707a1 and cyp707a2 mutants after Pst hrcC $^{-}$treatment (Additional file 1: Fig. S1d, e). Taken together, the above results indicate that CYP707A1 and CYP707A2 positively regulate plant responses to flg22 treatment.

\section{CYP707As are required for disease resistance to Pst}

There are four members of CYP707A genes in Arabidopsis genome, namely CYP707A1, CYP707A2, CYP707A3 and $C Y P 707 A 4$. The transcription levels of all four CYP707As were induced by dehydration and exogenous ABA treatment (Saito et al. 2004). We checked the expression patterns of these four genes in Plant eFP database. The results showed that all of the genes are induced by abiotic stress, such as auxin (IAA), ABA, methyl jasmonate (MeJA), cold, osmotic, salt and drought treatment. CYP707A1 and CYP707A4 transcription can also be induced by heat treatment (Additional file 1: Fig. S2a, b). For biotic stress, four genes showed reduced expression after flg22, Pst hrcC $^{-}$(a T3SS deficient mutant) and Pst treatment, except for CYP707A1 and CYP707A4 which, by contrast, were induced by Pst (Additional file 1: Fig. S2c). To confirm the results from the database, we used RT-qPCR to analyze the expression levels of CYP707A1 and CYP707A2, and found that both genes can be slightly induced by flg22 and Pst compared with mock treatment at $6 \mathrm{hpi}$, but returned to the base level at 24 hpi (Fig. 2a, b). In addition, cyp707a2 displayed enhanced susceptibility to Pst $\mathrm{hrcC}^{-}$and was more susceptible to Pst inoculation; however, cyp707a1 was more susceptible to Pst treatment but not Pst $\mathrm{hrcC}^{-}$(Fig. 2c, d). These data reveal that CYP707A1 and CYP707A2 are positive regulators of plant disease resistance to Pst, and suggest that Pst effector(s) likely targets CYP707s to promote pathogenicity.

\section{AvrPtoB interacts with CYP707As}

As the effector(s) may interfere with CYP707A-mediated immune response, we then investigated the potential 

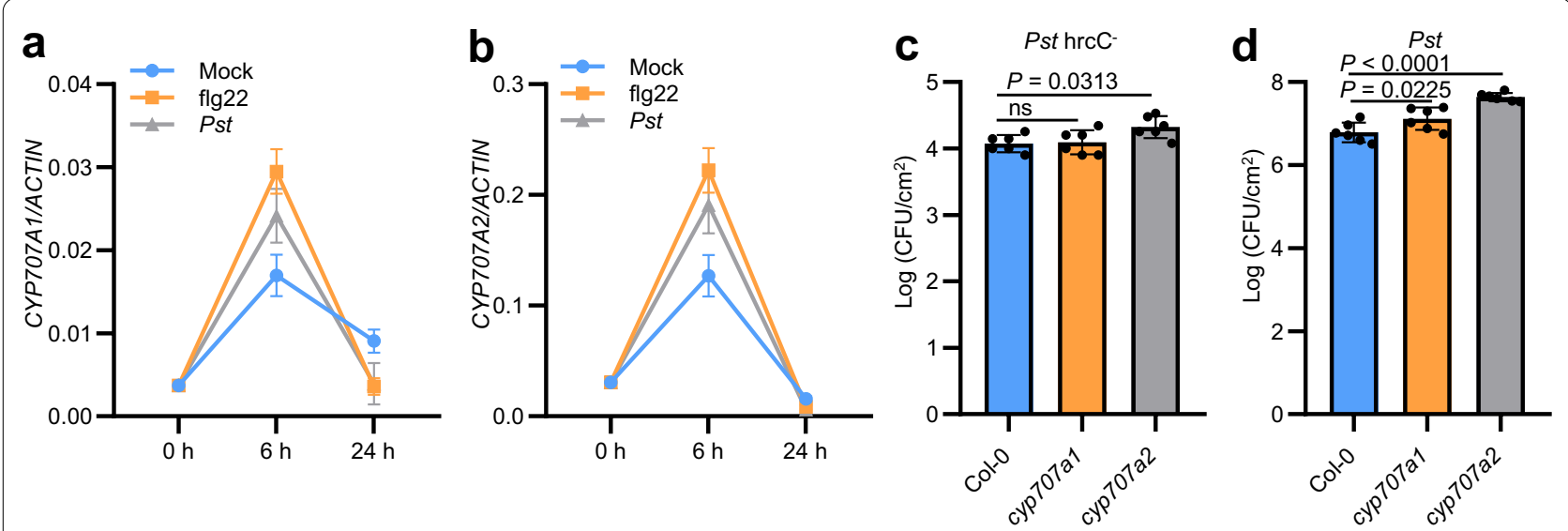

Fig. 2 CYP707As positively regulate disease resistance to Pst. a and $\mathbf{b}$ CYC707As expression after flg22 and Pst treatment. Four-week-old Arabidopsis leaves were inoculated with Mock (10 mM MgCl 2$), 100 \mathrm{nM}$ flg22 and Pst at a concentration of $5 \times 10^{7} \mathrm{CFU} / \mathrm{mL}$. Samples were collected for RT-qPCR at indicated time. Error bars represent means \pm SD ( $n=3$ biological replicates). $\mathbf{c}$ and $\mathbf{d}$ Growth of Pst hrcC ${ }^{-}$and Pst in Col-0 and cya707a mutants. Four-week-old Arabidopsis leaves were inoculated with Pst hrcC ${ }^{-}$(c) or Pst (d) at a concentration of $5 \times 10^{4} \mathrm{CFU} / \mathrm{mL}$. The plants were subjected to growth curve analysis at 3 days post-inoculation (dpi). Error bars represent means \pm SD (Two-way ANOVA, ns, no significance; $n=6$ )

\section{a}

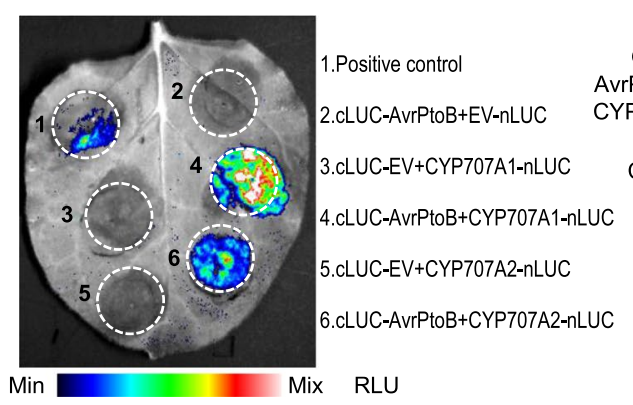

b



C

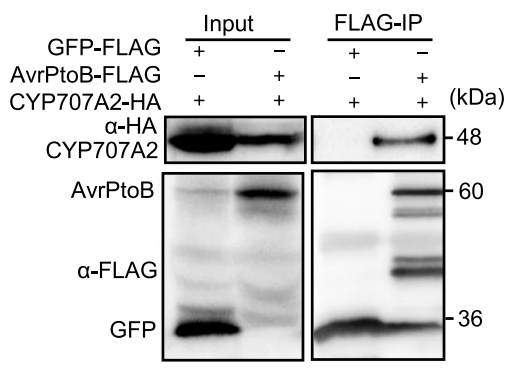

Fig. 3 AvrPtoB interacts with CYP707As. a AvrPtoB interacts with CYP707As in N. benthamiana by split luciferase assays. N. benthamiana leaves were co-infiltrated with 35S:CLUC-AvrPtoB and 35S:CYP707A1-nLUC or 355:CYP707A2-nLUC. Luciferase complementation imaging assays were performed $48 \mathrm{~h}$ later. This experiment was repeated three times with similar results. RLU, Relative light units. The combination of 35S:C2-nLUC and 35S:CLUC-S1 was used as a positive control. $\mathbf{b}$ and $\mathbf{c}$ AvrPtoB interacts with CYP707As in vivo by co-immunoprecipitation assays. N. benthamiana leaves were co-infiltrated with 355:CYP707A7-HA or 35S:CYP707A2-HA and 35S:AvrPtoB-FLAG or 35S:GFP-FLAG. Plant leaves were immunoprecipitated with anti-FLAG beads, and the proteins were immunoblotted by anti-FLAG antibody. Co-IP proteins were immunoblotted by anti-HA antibody

effector(s) in this event. Pst secretes ca. 30 effectors into plant cells (Xin and He 2013). In order to find the effector(s) that may target CYP707A1 or CYP707A2, we cloned all the effectors and screened them by spilt luciferase assays. The result showed that AvrPtoB interacted with both CYP707A1 and CYP707A2 (Fig. 3a). Subcellular localization assays showed that CYP707A1 and CYP707A2 were co-localized with the plasma membrane marker LTI6b-mCherry (Additional file 1: Fig. S3a, b), and exhibited the same localization pattern as AvrPtoB (Xu et al. 2020). To verify the interaction of AvrPtoB with CYP707A1 in vitro, we expressed the proteins in Escherichia coli and purified the recombinant proteins by affinity purification. The result showed that MBP-CYP707A1 successfully pulled down GST-AvrPtoB (Additional file 1: Fig. S4a). In addition, in anti-FLAG co-immunoprecipitation (Co-IP) assays, CYP707A1 and CYP707A2 interacted with AvrPtoB but not GFP alone in $N$. benthamiana leaves (Fig. 3b, c).

\section{AvrPtoB targets CYP707As for degradation}

The above results showed that AvrPtoB interacted with CYP707A1 and CYP707A2 in vitro and in vivo. We therefore explored the biological significance of the interactions. AvrPtoB is a 553 -amino-acid protein. Its $\mathrm{N}$-terminus and $\mathrm{C}$-terminus contain a Pto-interacting 

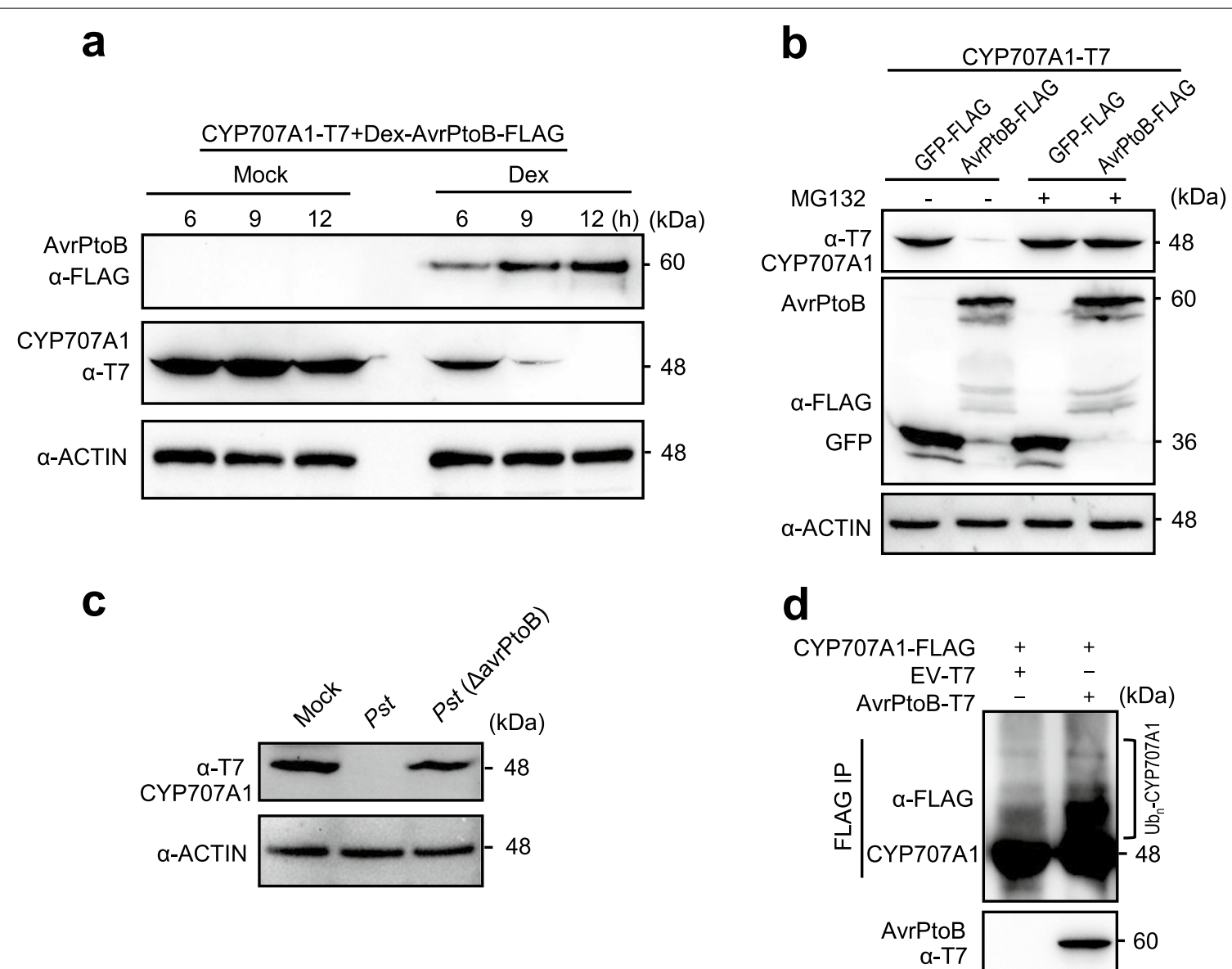

Fig. 4 AvrPtoB mediates CYP707A1 degradation by 265 proteasome. a AvrPtoB targets CYP707A1 for degradation. N. benthamiana leaves were co-infiltrated with 35S:CYP707A1-T7 and Dex:AvrPtoB-FLAG. Plants were infiltrated with $3 \mu \mathrm{M}$ Dex at 48 hpi to induce the expression of AvrPtoB-FLAG. Leaf extracts were sampled for immunoblotting after mock or Dex treatment at indicated times. Mock is $10 \mathrm{mM} \mathrm{MgCl} 2 . \mathbf{b}$ The proteasome inhibitor MG132 prevents CYP707A1 from degradation. 35S:CYP707A1-T7 was transiently expressed with 35S:GFP-FLAG or 35S:AvrPtoB-FLAG in N. benthamiana. MG132 (100 $\mu \mathrm{M})$ was used to inhibit 26S proteasome-mediated protein degradation at $36 \mathrm{hpi}$. Samples were harvested at $8 \mathrm{~h}$ after MG132 treatment for immunoblotting. $\mathbf{c}$ Pst harbored AvrPtoB degrades CYP707A1. 35S:CYP707A1-T7 stable transgenic plants were inoculated with Mock $\left(10 \mathrm{mM} \mathrm{MgCl}\right.$ ), Pst or Pst ( $\triangle$ avrPtoB) at a concentration of $2.5 \times 10^{3} \mathrm{CFU} / \mathrm{mL}$, respectively. Infected leaves were sampled for immunoblotting at 12 hpi. d AvrPtoB ubiquitinates CYP707A1 in vivo. 35S:CYP707A1-FLAG was transiently expressed with 35S:AvrPtoB-T7 or 35S:EV-T7 in N. benthamiana. MG132 (100 MM) was used to inhibit 26S proteasome-mediated protein degradation at $36 \mathrm{hpi}$. Samples were harvested at $8 \mathrm{~h}$ after MG132 treatment. Plant leaves were immunoprecipitated with anti-FLAG beads, and the proteins were immunoblotted by anti-FLAG and anti-T7 antibodies

domain (PID) and a U-box type E3 ubiquitin ligase domain, respectively (Janjusevic et al. 2006; Xiao et al. 2007). We further detected whether AvrPtoB can also mediate CYP707As degradation. In $N$. benthamiana leaves co-expressing dexamethasone (Dex)-inducible AvrPtoB-FLAG and 35S:CYP707A1-T7, Dex treatment significantly reduced the CYP707A1-T7 protein levels (Fig. 4a). However, CYP707A1-T7 protein levels were completely rescued in the presence of the $26 \mathrm{~S}$ proteasome inhibitor MG132 (Fig. 4b). To examine the degradation event during Pst infection, we inoculated the 35S:CYP707A1-T7 transgenic plant with Pst and Pst ( $\triangle$ avrPtoB). The result revealed that Pst rather than
Pst ( $\triangle$ avrPtoB) infection led to the degradation of CYP707A1 (Fig. 4c).

To assess whether AvrPtoB can degrade other CYP707As and key regulators in ABA signaling, we also detected their protein levels when co-expressed with AvrPtoB in $N$. benthamiana leaves. As shown in Additional file 1: Fig. S4b, CYP707A3 protein level was reduced when co-expressed with AvrPtoB, the same as that for CYP707A1 and CYP707A2. But the PP2Cs ABI1 and $A B I 2$, two negative regulators of $A B A$ signaling pathway, showed no significant differences when co-expressed with AvrPtoB or GFP alone (Additional file 1: Fig. S4c). NGATHA (NGA1) is a transcriptional activator of the 
key enzyme NINECIS-EPOXYCAROTENOID DIOXYGENASE 3 (NCED3) in ABA biosynthesis (Sato et al. 2018). The protein level of NGATHA was not affected by AvrPtoB, neither was VirE2-INTERACTING PROTEIN1 (VIP1), a transcriptional activator of CYP707A1 and CYP707A3 (Additional file 1: Fig. S4d) (Tsugama et al. 2012). To investigate whether AvrPtoB can ubiquitinate CYP707As in vivo, we co-expressed CYP707As and AvrPtoB in N. benthamiana. By FLAG Co-IP assays, we found that CYP707A1 and CYP707A2 were highly ubiquitinated when co-expressed with AvrPtoB but not with EV (Fig. 4d and Additional file 1: Fig. S4e). In summary, these results demonstrate that CYP707As are the target of AvrPtoB and can be degraded via 26S proteasome.

\section{AvrPtoB promotes ABA sensitivity in Arabidopsis}

Previous studies have demonstrated that AvrPtoB transgenic seedlings are hypersensitive to SA-induced toxicity and Dex:HopAM1 transgenic lines are severely inhibited by ABA (Goel et al. 2008; Chen et al. 2017). Dex:AvrPtoB transgenic plant can induce a significant increase in ABA levels after Dex treatment for $6 \mathrm{~h}$ (de Torres-Zabala et al. 2007). We therefore examined the responses of the $p E s t: A v r P t o B$ transgenic seedlings in the presence of ABA. The two AvrPtoB transgenic lines exhibited a lower cotyledon greening rate than Col-0 and $p E s t: E V$ (empty vector) transgenic line (Fig. $5 \mathrm{a}-\mathrm{c}$ ). AvrPtoB also markedly induced the expression of NCED 3 and RAB18, two ABAresponsive genes (Fig. 5d, e). Furthermore, we determined whether AvrPtoB can also regulate auxin and JA signaling pathways. AvrPtoB transgenic seedlings showed no significant difference in seed germination compared with Col-0 and $p E s t: E V$ transgenic lines after auxin (IAA), methyl jasmonate (MeJA) and extradiol treatment (Additional file 1: Fig. S5a, b), but they exhibited hypersensitivity to SA treatment (Additional file 1: Fig. S5c) (Chen et al. 2017). Nevertheless, these results indicate that AvrPtoB promotes sensitivity to ABA in Arabidopsis.

\section{CYP707As are virulent targets of AvrPtoB}

To gain insight into the function of CYP707As in plant immunity, we next analyzed the role of AvrPtoB in CYP707As-mediated defense during Pst infection. We compared bacterial replication in Col-0, cyp707a1 and cyp707a2 after inoculation with Pst or Pst ( $\triangle \mathrm{avrPtoB})$. There was no significant difference between Col-0 and cyp707a1 under Pst ( $\triangle$ avrPtoB) treatment, while the difference between Col-0 and cyp707a2 was reduced when compared to Pst treatment (Fig. 6a). Exogenous application of ABA treatment resulted in the proliferation of Pst. We found that when the plants were pre-treated with $\mathrm{ABA}$ or $\mathrm{ABA}$ inhibitor fluridone, Pst proliferated to a similar level in Col-0 and cyp707a1; however, the difference between Col-0 and cyp707a2 was significantly reduced than mock treatment (Fig. 6b, c). Notably, fluridone did not inhibit the growth of Pst (Fig. 6d). These results further suggest that AvrPtoB targets CYP707A1 and CYP707A2 to promote infection.

\section{Discussion}

$\mathrm{ABA}$ is a major phytohormone that is involved in a variety of biotic and abiotic responses in plants. Although ABA has been demonstrated to have a clear role in abiotic stresses, it remains disputed for its roles in plant immunity (Adie et al. 2007; Hernández-Blanco et al. 2007; Fan et al. 2009; Cao et al. 2011; Xiao et al. 2017; Tan et al. 2019). ABA induces stomatal closure under drought stress, and this prevents plants from water loss. It is known that stomatal closure can prevent $P s t$ invasion through these natural pores (Melotto et al. 2006). However, during post-invasive stage, stomatal closure facilitates the establishment of an aqueous intercellular space with high humidity, which benefits $P$ st proliferation (Xin et al. 2016).

In addition to manipulating stomata to help prevent water loss, endogenous ABA has been found to facilitate Pst infection. In fact, Pst infection could induce ABA accumulation in Arabidopsis, and the effector protein AvrPtoB has been suggested to dictate this process (de Torres-Zabala et al. 2007). By screening the Arabidopsis mutant stock, we discovered that cyp707a1 and cyp707a2 mutants were susceptible to Pst infection, and identified CYP707A proteins as the targets of AvrPtoB to induce ABA accumulation. Therefore, we resolved the mystery of Pst-induced ABA accumulation in Arabidopsis (Fig. 7). CYP707As are key enzymes in the oxidative catabolism of ABA and their roles in plant immunity are unclear (Kushiro et al. 2004; Saito et al. 2004). ABA can attenuate callose deposition which is associated with basal defense (de Torres-Zabala et al. 2007; GarcíaAndrade et al. 2011). ABA pre-treatment can reduce flg22-induced $\mathrm{H}_{2} \mathrm{O}_{2}$ generation (Tan et al. 2019). The reduced production of flg22-induced ROS in cyp $707 a$ mutants may be attributed to the high level of endogenous ABA. Although only the cyp707a2 mutant showed enhanced susceptibility to Pst hrcC $\mathrm{C}^{-}$, both cyp707a1 and cyp707a2 mutants were susceptible to Pst (Fig. 2), highlighting the CYP707As' role in plant basal defense.

By in vitro and in vivo protein-protein interaction assays, we were able to show that CYP707A1 and CYP707A2 physically interacted with AvrPtoB. AvrPtoB is a C-terminal U-box type E3 ubiquitin ligase and targets multiple immune regulators in host cells, such as FLS2, CERK1, LecRK-IX.2 and NPR1 (Göhre et al. 2008; Gimenez-Ibanez et al. 2009; Chen et al. 2017; Xu et al. 2020). Unlike the immune regulators, CYP707 family proteins 


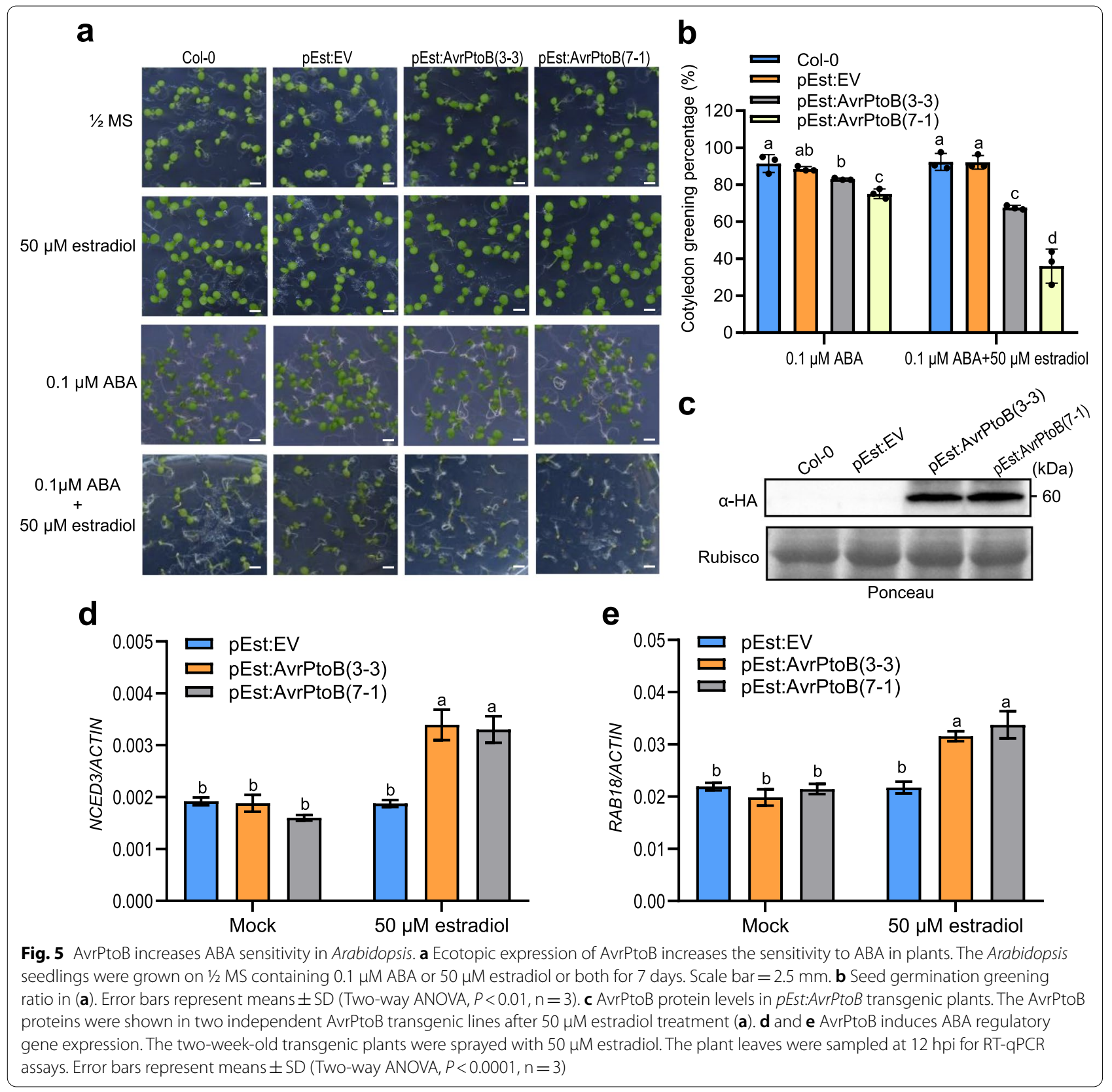

have not been shown to act in plant immune responses yet. CYP707A proteins contribute to ROS burst and callose deposition during pathogen infection, indicating their roles in early immune responses (Fig. 1). However, CYP707As are key enzymes that catalyze ABA to an inactive form. The cyp $707 a$ mutants accumulated high levels of endogenous ABA and were susceptible to Pst, suggesting that CYP707A could inactivate ABA to attenuate Pst infection. AvrPtoB can increase the expression of NCED3 and foliar ABA levels in Arabidopsis, however, it is unknown how AvrPtoB manipulates plant ABA signaling pathway (de Torres-Zabala et al. 2007). In this article, we revealed that CYP707A1 and CYP707A2 are additional targets of AvrPtoB.

It has been reported that many effectors promote pathogenicity through manipulating plant hormone signaling pathway. HopAM1 is the first type III effector that was reported to aid pathogen adaptation to water availability in plant. Although the expression of HopAM1 in transgenic plants does not induce ABA production, it does enhance ABA responses and suppress basal defenses (Goel et al. 2008). HopZa1 targets the orthologues of 

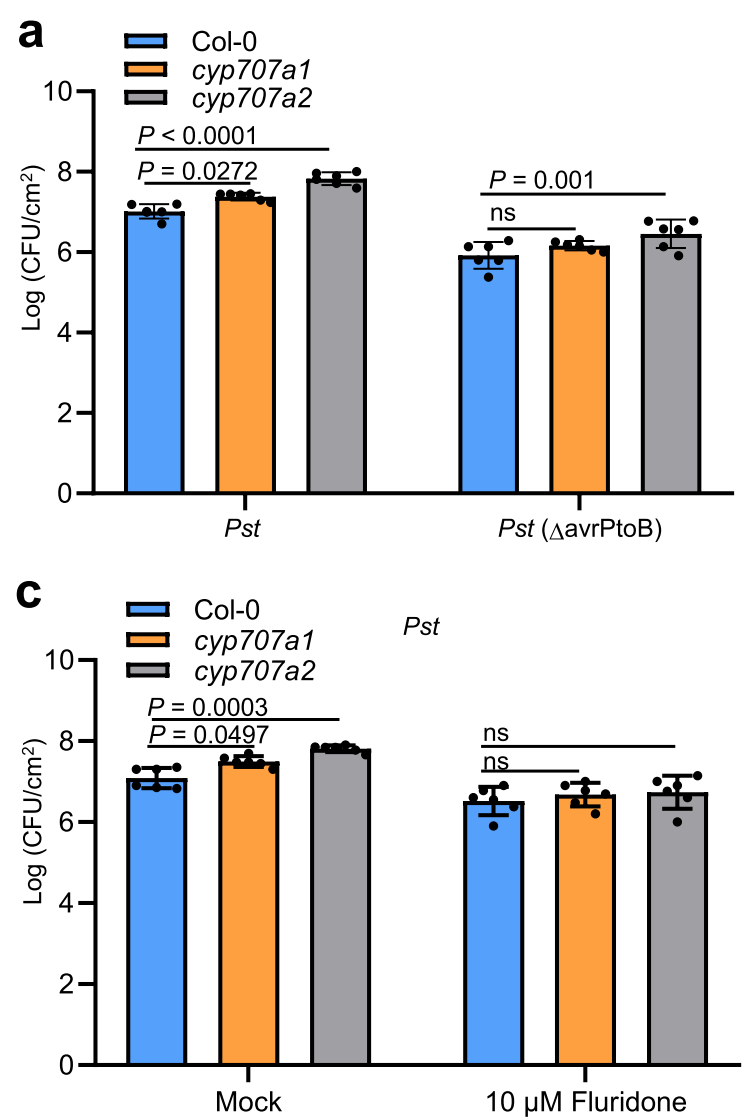

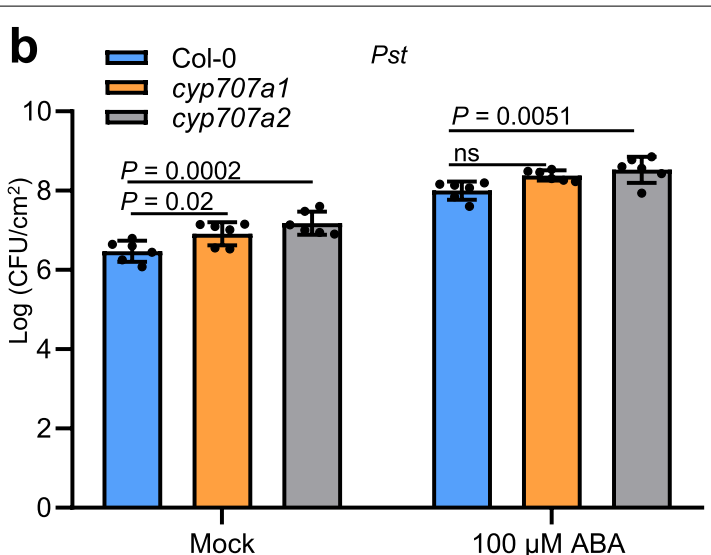

d

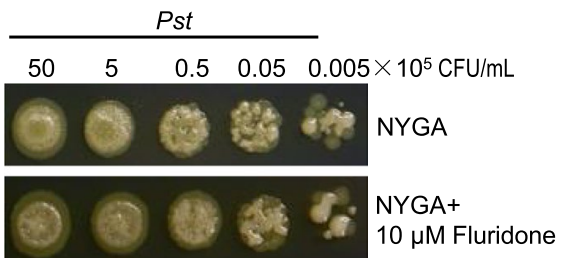

Fig. 6 CYP707As are virulent targets of AvrPtoB. a-c Growth of Pst and Pst ( $\triangle$ avrPtoB) in Col-0 and cya707a mutants. Four-week-old Arabidopsis leaves were inoculated with Pst and Pst ( $\triangle$ avrPtoB) at a concentration of $5 \times 10^{4} \mathrm{CFU} / \mathrm{mL}(\mathbf{a})$ or sprayed with Mock $\left(\mathrm{H}_{2} \mathrm{O}\right)$ or $\mathrm{ABA}(100 \mu \mathrm{M})(\mathbf{b})$ or $10 \mu \mathrm{M}$ fluridone (c) $12 \mathrm{~h}$ before pathogen treatment. The plants were subjected to growth curve analysis at $3 \mathrm{dpi}$. Error bars represent means \pm SD (Two-way ANOVA, ns, no significance; $\mathrm{n}=6$ ). $\mathbf{d} 10 \mu \mathrm{M}$ fluridone doesn't inhibit Pst growth on NYGA medium. The Pst was serially diluted on NYGA medium with or without $10 \mu \mathrm{m}$ fluridone. The photographs were taken after incubation for 4 days

JAZ1 in both soybean (Glycine max) and Arabidopsis to promote their degradation in a COI1-dependent manner, thereby activating JA signaling to enhance Pst infection (Jiang et al. 2013). For the hemi-biotrophic fungus Fusarium oxysporum, the effector SECRETED IN XYLEM4 (FoSIX4) can contribute to disease development caused by $F$ oxysporum when expressed in Arabidopsis. Arabidopsis plants inoculated with the six4 mutant strain show reduced expressions of JA-responsive genes, demonstrating that FoSIX4 promotes pathogen virulence via activating host JA signaling pathway (Thatcher et al. 2012). In addition to JA, ethylene is a gaseous hormone that regulates various biological processes in plants, including defense against pathogens. The Xanthomonas euvesicatoria (Xcv) effector protein XopD, carrying a C-terminal SUMO protease domain, is reported to target the tomato ethylene responsive transcription factor SIERF4 to suppress ethylene production, which is required for anti-Xcv immunity and symptom development (Kim et al. 2013).
HopAF1 secreted by P.syringae inhibits host defense response by manipulating MTN (methylthioadenosine nucleosidase) activity and consequently dampens ethylene production (Washington et al. 2016). As a counterdefense strategy, oomycetes pathogen Phytophthora sojae secretes the RXLR effector PsAvh238 to destabilize plant Type2 1-aminocyclopropane-1-carboxylate synthases (ACSs), the key enzymes in catalyzing the rate-limiting step of ET biosynthesis, to reduce ET production and promote infection (Yang et al. 2019).

\section{Conclusions}

Taken together, we discovered an additional virulence target of the Pst effector AvrPtoB in Arabidopsis. We demonstrated that AvrPtoB induced ABA accumulation by degrading ABA 8'-Hydroxylase CYP707As to promote Pst infection. Because AvrPtoB targets multiple proteins in plants, it is interesting to unravel the dynamic interactions of AvrPtoB with these proteins in future studies. 


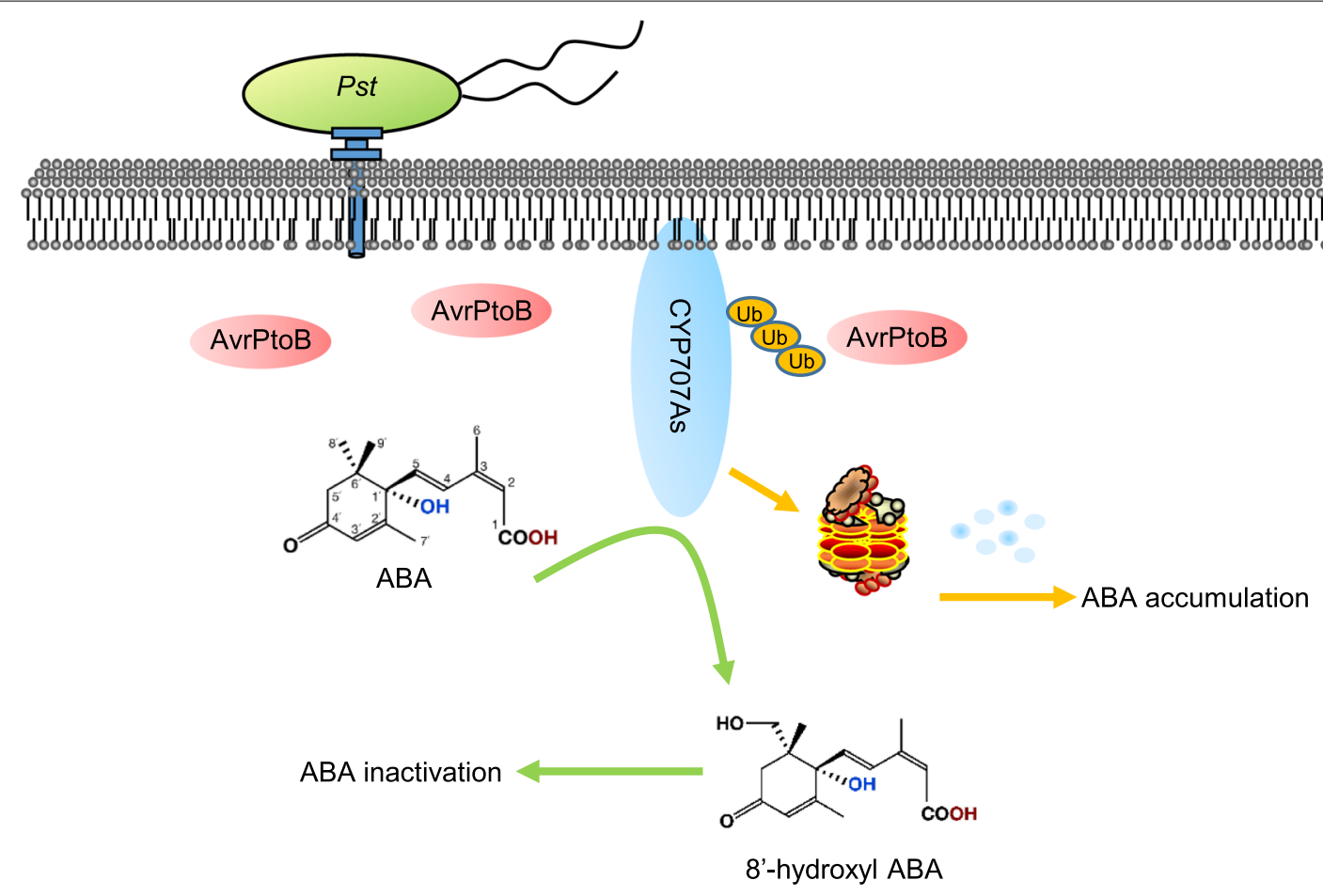

Fig. 7 The working model. ABA is catalyzed to 8'-hydroxyl ABA by CYP707As, a group of P450-type mono-oxygenases, to maintain ABA signal homeostasis in Arabidopsis plants. During Pst infection, the pathogen-delivered effector AvrPtoB degrades CYP707As via 265 proteasome. As a result, $A B A$ is accumulated, which leads to increased susceptibility to Pst

In addition, how $\mathrm{ABA}$ enhances plant susceptibility to Pst is still unknown. It is worth investigating if ABA could increase interior humidity in plant cells, thereby facilitating pathogen proliferation.

\section{Methods}

\section{Plant materials and growth conditions}

A. thaliana T-DNA insertion mutants cyp707a1-1 (SALK_069127) and cyp707a2-2 (SALK_083966c) were used. Plants were grown at $23{ }^{\circ} \mathrm{C}$ under $10 \mathrm{~h}$ of light/14 h of dark for 4 weeks. $p E s t: E V, p E s t: A v r P t o B$ and 35S:CYP707A1-T7 transgenic plants were generated via floral dip transformation procedure (Clough and Bent 1998). For phytohormones phenotyping assays, surfacesterilized seeds were sowed on 1/2 MS medium with or without phytohormones or estradiol. The seeds were stratified at $4{ }^{\circ} \mathrm{C}$ for 3 days in dark before being planted on media. Then the plates were moved to a growth chamber at $23^{\circ} \mathrm{C}$ under short-day conditions.

\section{ROS burst measurement}

Three-week-old Arabidopsis seedling leaves were sampled for leaf disks and kept in 96-well plate with $\mathrm{ddH}_{2} \mathrm{O}$ overnight. Before measurement, $\mathrm{dd}_{2} \mathrm{O}$ was replaced by reaction mixtures containing $17 \mathrm{mM}$ luminol L-012
(Wako), $10 \mathrm{mg} / \mathrm{mL}$ horseradish peroxidase, $100 \mathrm{nM}$ flg22 or $100 \mathrm{nM}$ elf18. Each treatment contained at least three replications. Luminescence was measured continuously for 30 min using Infinite F200 (TECAN).

\section{MAPK assay}

Two-week-old Arabidopsis seedlings on the plate were sprayed with $\mathrm{ddH}_{2} \mathrm{O}$ or $100 \mathrm{nM}$ flg22. Samples were collected and frozen in liquid nitrogen at indicated time points. The total proteins were extracted with $1 \times$ laemmli $(0.0625 \mathrm{M}$ Tris-HCl, 10\% glycerol, 2\% SDS, 0.0025\% bromophenol blue, 5\% 2-mercaptoethanol, $\mathrm{pH}$ 6.8) buffer and separated on a 12\% SDS-PAGE. Activated MAPKs were detected by immunoblotting with phospho-p44/42 MAPK antibody (Cell Signaling).

\section{Callose staining and quantification}

Leaves of four-week-old Arabidopsis plants were infiltrated with $\mathrm{H}_{2} \mathrm{O}, 100 \mathrm{nM}$ flg22 or Pst $\mathrm{hrCC}^{-}$at a concentration of $5 \times 10^{7} \mathrm{CFU} / \mathrm{mL}$ in $10 \mathrm{mM}$ of $\mathrm{MgCl}_{2}$ for $12 \mathrm{~h}$ or $24 \mathrm{~h}$. The leaves were transferred into FAA solution ( $10 \%$ formaldehyde, $5 \%$ acetic acid and $50 \%$ ethanol) for $12 \mathrm{~h}$, de-stained in $95 \%$ ethanol for $6 \mathrm{~h}$ and washed twice with $\mathrm{ddH}_{2} \mathrm{O}$, then stained with $0.01 \%$ aniline blue in $150 \mathrm{mM} \mathrm{KH}_{2} \mathrm{PO} 4(\mathrm{pH} 9.5)$ for $1 \mathrm{~h}$ at room temperature. 
The callose deposits were visualized with a fluorescence microscope (OLYMPUS IX71). Callose deposits were counted by Image J software.

\section{RT-qPCR}

Total RNA was isolated from plants treated with different conditions at indicated time points by TRIzol Reagent (Invitrogen) according to the technical manual. One microgram of total RNA was subjected to synthesize the first-strand cDNA by HiScript Q RT SuperMix with a genomic DNA wipe (Vazyme, China) according to the technical manual. qPCR was performed by the Bio-Rad system using ChamQ SYBR qPCR Master Mix (Vazyme, China). Actin 2 was used as an internal control. Each sample was performed in triplicate (Additional file 2: Table S1).

\section{Pathogen inoculation assay}

Bacterial strains were grown on NYGA medium $(0.5 \%$ Peptone, $0.3 \%$ yeast extract and $0.2 \%$ glycerin) at $28{ }^{\circ} \mathrm{C}$. Four-week-old Col-0, cyp707a1 and cyp707a2 were infiltrated with Pst, Pst $\mathrm{hrcC}^{-}$or Pst ( $\triangle$ avrPtoB) at a concentration of $5 \times 10^{4} \mathrm{CFU} / \mathrm{mL}$, respectively. Three days after inoculation, plants were subjected to growth curve analysis as described by Liu (Liu et al. 2011). The Pst $(\Delta$ avrPtoB) deletion mutant were described previously (Xu et al. 2020).

\section{Transient expression in Nicotiana benthamiana}

For split-luciferase complementation assay, Agrobacterium tumefaciens (strain EHA105) carrying the indicated nLUC and cLUC constructs was mixed and infiltrated into 4-week-old $N$. benthamiana leaves. 35S:C2-nLUC and 35S:cLUC-S1 were used as a positive control (Zhang et al. 2011). Two days after infiltration, $N$. benthamiana leaves were rubbed with $0.5 \mathrm{mM}$ D-luciferin (Gold Biotechnology) and kept in the dark for $5 \mathrm{~min}$. The luciferase images were captured by Tanon-5200 (Chen et al. 2008).

For subcellular localization assay, CYP707A1 or CYP707A2 were fused to GFP at their C-terminal under the control of $35 \mathrm{~S}$ promoter (35S:CYP707A1-GFP or 35S:CYP707A2-GFP) and transiently expressed in $N$. benthamiana. The images were observed using a Leica SP8 confocal laser microscope at 48 hpi. LTI6b-mCherry was used as a marker.

For Co-IP assay, CYP707A1-HA, CYP707A2-HA, GFPFLAG and AvrPtoB-FLAG under the control of $35 \mathrm{~S}$ promoter were transient expressed in $N$. benthamiana by $A$. tumefaciens strain GV3101. At about $48 \mathrm{~h}$, the infiltrated leaves were sampled and total proteins were extracted with extraction buffer $(50 \mathrm{mM}$ Tris- $\mathrm{HCl}, 150 \mathrm{mM} \mathrm{NaCl}$, 0.1\% Triton, $0.2 \%$ NP-40, $6 \mathrm{mM}$ 2-mercapto-Ethanol and proteinase inhibitor cocktail (Roche), pH7.5). The
anti-FLAG IP was performed by incubating the proteins with $30 \mu \mathrm{L}$ anti-FLAG (R) M2 Affinity Gel (SigmaAldrich, catalog \# A2220) for $2 \mathrm{~h}$ on an end-over-end shaker at $4{ }^{\circ} \mathrm{C}$. After washing three times with extraction buffer, the eluted proteins were separated by SDS-PAGE and revealed by immunoblot analysis using anti-FLAG and anti-HA antibody.

\section{Recombinant protein purification}

GST-AvrPtoB were purified as described previously (Xu et al. 2020). CYP707A1 were cloned into the vector pMal-C4X. The positive clones were transformed into Escherichia coli (BL21). Bacterial cells were grown in Luria Broth (LB) medium at $37{ }^{\circ} \mathrm{C}$ with shaking until the OD600 reaches 0.6. The MBP-CYP707A1 was induced with $0.5 \mathrm{mM}$ IPTG at $16{ }^{\circ} \mathrm{C}$ overnight and purified using amylose beads according to the technical manual. The purified proteins were ultrafiltrated and diluted in PBS buffer containing $10 \%$ glycerin to $1 \mu \mathrm{g} / \mu \mathrm{L}$ and stored at $-80{ }^{\circ} \mathrm{C}$ before use.

\section{MBP pull-down assays}

MBP pull-down assays was performed as described by Liu et al. (2011) with minor modification. In brief, $3 \mu \mathrm{g}$ of each MBP-CYP707A1 and GST-AvrPtoB were incubated in TEN100 buffer (20 mM Tris- $\mathrm{HCl}$ (pH 7.4), $100 \mathrm{mM}$ $\mathrm{NaCl}, 0.1 \mathrm{mM}$ EDTA and $0.2 \%$ Triton X-100) with 30 $\mu \mathrm{L}$ amylose beads on an earthquake shaker for $2 \mathrm{~h}$ at $4{ }^{\circ} \mathrm{C}$. Then the beads were washed at least 4 times with NETN300 buffer (20 mM Tris- $\mathrm{HCl}$ (pH 7.4), $300 \mathrm{mM}$ $\mathrm{NaCl}, 0.1 \mathrm{mM}$ EDTA and $0.5 \% \mathrm{NP}-40$ ). The proteins were eluted by adding $50 \mu \mathrm{L} 1 \times$ laemmli buffer and boiled for $5 \mathrm{~min}$ at $95{ }^{\circ} \mathrm{C}$. Eluted proteins were separated on a $12 \%$ SDS-PAGE gel and immunoblotted with anti-MBP and anti-GST antibody, respectively.

\section{Abbreviations \\ ABA: Abscisic acid; Co-IP: Co-immunoprecipitation; Dex: Dexamethasone; flg22: A 22-amino-acid peptide of bacterial flagellin; GST: Glutathione S-transferase; IAA: 3-Indoleacetic acid; JA: Jasmonic acids; MAMPs: Microbe- associated molecular patterns; MBP: Maltose-binding protein; MeJA: Methyl Jasmonate; MTN: Methylthioadenosine nucleosidase; MS: Murashige and Skoog; PA: Phaseic acid; PAMPs: Pathogen-associated molecular patterns: PP2Cs: Phosphatase 2Cs; RT-qPCR: Reverse transcription quantitative PCR; ROS: Reactive oxygen species; SA: Salicylic acid; T3SS: Type III protein secretion system.}

\section{Supplementary Information}

The online version contains supplementary material available at https://doi. org/10.1186/s42483-022-00110-8.

Additional file 1: Figure S1. The cyp707a mutants demonstrates defective PTI response. Figure S2. Expression profiles of CYP707A1, CYP707A2, CYP707A3 and CYP707A4 in response to biotic stress, abiotic stress and 
hormone treatment. Figure S3. Subcellular localization of CYP707A1 and CYP707A2. Figure S4. AvrPtoB targets CYP707As for degradation. Figure S5. AvrPtoB does not interfere with IAA, SA and MeJA signaling pathways.

Additional file 2: Table S1. Primers used in this study.

\section{Acknowledgements}

We thank Prof. Qi Xie from Institute of Genetics and Developmental Biology in Chinese Academy of Sciences and Prof. Kai Shu from Northwestern Polytechnical University for kindly providing us the mutant seeds cyp707a1 and cyp707a2.

\section{Authors' contributions}

$J L$ and NX conceived and designed the project. YL and NX performed the experiments and analyzed the data. JL, NX, YL, and M. R. Mahmud wrote the article. JL supervised research. All authors read and approved the final manuscript.

\section{Funding}

This work was supported by the National Natural Science Foundation of China (32070290).

\section{Availability of data and materials}

Not applicable.

\section{Declarations}

Ethical approval and consent to participate

Not applicable.

\section{Consent for publication}

Not applicable.

\section{Competing interests}

The authors declare that they have no competing interests.

\section{Author details}

'State Key Laboratory of Plant Genomics, Institute of Microbiology, Chinese Academy of Sciences, Beijing 100101, China. ${ }^{2}$ University of the Chinese Academy of Sciences, Beijing, China. ${ }^{3}$ Department of Plant Pathology, MOA Key Lab of Pest Monitoring and Green Management, College of Plant Protection, China Agricultural University, Beijing 100193, China. ${ }^{4}$ Department of Plant Pathology, Patuakhali Science and Technology University, Patuakhali 8602, Bangladesh.

\section{Received: 17 January 2022 Accepted: 18 February 2022}

Published online: 01 March 2022

\section{References}

Adie BA, Pérez-Pérez J, Pérez-Pérez MM, Godoy M, Sánchez-Serrano JJ, Schmelz $E A$, et al. ABA is an essential signal for plant resistance to pathogens affecting JA biosynthesis and the activation of defenses in Arabidopsis. Plant Cell. 2007;19:1665-81. https://doi.org/10.1105/tpc.106.048041.

Asai T, Tena G, Plotnikova J, Willmann MR, Chiu WL, Gomez-Gomez L, et al. MAP kinase signalling cascade in Arabidopsis innate immunity. Nature. 2002:415:977-83. https://doi.org/10.1038/415977a.

Brandt B, Brodsky DE, Xue S, Negi J, Iba K, Kangasjärvi J, et al. Reconstitution of abscisic acid activation of SLAC1 anion channel by CPK6 and OST1 kinases and branched ABI1 PP2C phosphatase action. Proc Natl Acad Sci U S A. 2012;109:10593-8. https://doi.org/10.1073/pnas.1116590109.

Cao FY, Yoshioka K, Desveaux D. The roles of ABA in plant-pathogen interactions. J Plant Res. 2011;124:489-99. https://doi.org/10.1007/ s10265-011-0409-y.

Chen H, Zou Y, Shang Y, Lin H, Wang Y, Cai R, et al. Firefly luciferase complementation imaging assay for protein-protein interactions in plants. Plant Physiol. 2008;146:368-76. https://doi.org/10.1104/pp.107.111740.

Chen H, Chen J, Li M, Chang M, Xu K, Shang Z, et al. A bacterial type III effector targets the master regulator of salicylic acid signaling, NPR1, to subvert plant immunity. Cell Host Microbe. 2017;22:777-88.e7. https://doi.org/10. 1016/j.chom.2017.10.019.

Chen K, Li GJ, Bressan RA, Song CP, Zhu JK, Zhao Y. Abscisic acid dynamics, signaling, and functions in plants. J Integr Plant Biol. 2020;62:25-54. https://doi.org/10.1111/jipb.12899.

Clough SJ, Bent AF. Floral dip: a simplified method for Agrobacteriummediated transformation of Arabidopsis thaliana. Plant J. 1998;16:735-43. https://doi.org/10.1046/j.1365-313x.1998.00343.x.

Cutler SR, Rodriguez PL, Finkelstein RR, Abrams SR. Abscisic acid: emergence of a core signaling network. Annu Rev Plant Biol. 2010;61:651-79. https:// doi.org/10.1146/annurev-arplant-042809-112122.

Dai M, Xue Q, McCray T, Margavage K, Chen F, Lee JH, et al. The PP6 phosphatase regulates $A B 15$ phosphorylation and abscisic acid signaling in Arabidopsis. Plant Cell. 2013;25:517-34. https://doi.org/10.1105/tpc.112. 105767.

de Torres-Zabala M, Truman W, Bennett MH, Lafforgue G, Mansfield JW, Rodriguez Egea P, et al. Pseudomonas syringae pv. tomato hijacks the Arabidopsis abscisic acid signalling pathway to cause disease. EMBO J. 2007;26:1434-43. https://doi.org/10.1038/sj.emboj.7601575.

Ding P, Ding Y. Stories of salicylic acid: a plant defense hormone. Trends Plant Sci. 2020;25:549-65. https://doi.org/10.1016/j.tplants.2020.01.004.

Fan J, Hill L, Crooks C, Doerner P, Lamb C. Abscisic acid has a key role in modulating diverse plant-pathogen interactions. Plant Physiol. 2009;150:175061. https://doi.org/10.1104/pp.109.137943.

Gao S, Guo W, Feng W, Liu L, Song X, Chen J, et al. LTP3 contributes to disease susceptibility in Arabidopsis by enhancing abscisic acid (ABA) biosynthesis. Mol Plant Pathol. 2016;17:412-26. https://doi.org/10.1111/mpp.12290.

García-Andrade J, Ramírez V, Flors V, Vera P. Arabidopsis ocp3 mutant reveals a mechanism linking $A B A$ and $J A$ to pathogen-induced callose deposition. Plant J. 2011;67:783-94. https://doi.org/10.1111/j.1365-313x.2011.04633.x.

García-Andrade J, González B, Gonzalez-Guzman M, Rodriguez PL, Vera P. The role of ABA in plant immunity is mediated through the PYR1 receptor. Int J Mol Sci. 2020. https://doi.org/10.3390/ijms21165852.

Gimenez-Ibanez S, Hann DR, Ntoukakis V, Petutschnig E, Lipka V, Rathjen JP. AvrPtoB targets the LysM receptor kinase CERK1 to promote bacterial virulence on plants. Curr Biol. 2009;19:423-9. https://doi.org/10.1016/j. cub.2009.01.054.

Goel AK, Lundberg D, Torres MA, Matthews R, Akimoto-Tomiyama C, Farmer L, et al. The Pseudomonas syringae type III effector HopAM1 enhances virulence on water-stressed plants. Mol Plant Microbe Interact. 2008;21:36170. https://doi.org/10.1094/mpmi-21-3-0361.

Göhre V, Spallek T, Häweker H, Mersmann S, Mentzel T, Boller T, et al. Plant pattern-recognition receptor FLS2 is directed for degradation by the bacterial ubiquitin ligase AvrPtoB. Curr Biol. 2008;18:1824-32. https://doi. org/10.1016/j.cub.2008.10.063.

Gómez-Gómez L, Felix G, Boller T. A single locus determines sensitivity to bacterial flagellin in Arabidopsis thaliana. Plant J. 1999;18:277-84. https:// doi.org/10.1046/j.1365-313x.1999.00451.x.

Hernández-Blanco C, Feng DX, Hu J, Sánchez-Vallet A, Deslandes L, Llorente $F$, et al. Impairment of cellulose synthases required for Arabidopsis secondary cell wall formation enhances disease resistance. Plant Cell. 2007;19:890-903. https://doi.org/10.1105/tpc.106.048058.

Janjusevic R, Abramovitch RB, Martin GB, Stebbins CE. A bacterial inhibitor of host programmed cell death defenses is an E3 ubiquitin ligase. Science. 2006;311:222-6. https://doi.org/10.1126/science.1120131.

Jiang S, Yao J, Ma KW, Zhou H, Song J, He SY, et al. Bacterial effector activates jasmonate signaling by directly targeting JAZ transcriptional repressors. PLoS Pathog. 2013;9: e1003715. https://doi.org/10.1371/journal.ppat. 1003715.

Kim JG, Stork W, Mudgett MB. Xanthomonas type III effector XopD desumoylates tomato transcription factor SIERF4 to suppress ethylene responses and promote pathogen growth. Cell Host Microbe. 2013;13:143-54. https://doi.org/10.1016/j.chom.2013.01.006.

Kunze G, Zipfel C, Robatzek S, Niehaus K, Boller T, Felix G. The N terminus of bacterial elongation factor Tu elicits innate immunity in Arabidopsis plants. Plant Cell. 2004;16:3496-507. https://doi.org/10.1105/tpc.104. 026765.

Kushiro T, Okamoto M, Nakabayashi K, Yamagishi K, Kitamura S, Asami T, et al. The Arabidopsis cytochrome P450 CYP707A encodes ABA 8'-hydroxylases: key enzymes in ABA catabolism. EMBO J. 2004;23:1647-56. https://doi. org/10.1038/sj.emboj.7600121. 
Li N, Han X, Feng D, Yuan D, Huang LJ. Signaling crosstalk between salicylic acid and ethylene/jasmonate in plant defense: do we understand what they are whispering? Int J Mol Sci. 2019. https://doi.org/10.3390/ijms2 0030671.

Liu J, Elmore JM, Lin ZJ, Coaker G. A receptor-like cytoplasmic kinase phosphorylates the host target RIN4, leading to the activation of a plant innate immune receptor. Cell Host Microbe. 2011;9:137-46. https://doi.org/10. 1016/j.chom.2011.01.010.

Ma Y, Szostkiewicz I, Korte A, Moes D, Yang Y, Christmann A, et al. Regulators of PP2C phosphatase activity function as abscisic acid sensors. Science. 2009;324:1064-8. https://doi.org/10.1126/science.1172408.

Melotto M, Underwood W, Koczan J, Nomura K, He SY. Plant stomata function in innate immunity against bacterial invasion. Cell. 2006;126:969-80. https://doi.org/10.1016/j.cell.2006.06.054.

Meyer K, Leube MP, Grill E. A protein phosphatase 2C involved in ABA signal transduction in Arabidopsis thaliana. Science. 1994;264:1452-5. https:// doi.org/10.1126/science.8197457.

Nambara E, Marion-Poll A. Abscisic acid biosynthesis and catabolism. Annu Rev Plant Biol. 2005;56:165-85. https://doi.org/10.1146/annurev.arplant. 56.032604.144046.

Okamoto M, Kuwahara A, Seo M, Kushiro T, Asami T, Hirai N, et al. CYP707A1 and CYP707A2, which encode abscisic acid 8'-hydroxylases, are indispensable for proper control of seed dormancy and germination in Arabidopsis. Plant Physiol. 2006;141:97-107. https://doi.org/10.1104/pp. 106.079475.

Saito S, Hirai N, Matsumoto C, Ohigashi H, Ohta D, Sakata K, et al. Arabidopsis CYP707As encode (+)-abscisic acid 8'-hydroxylase, a key enzyme in the oxidative catabolism of abscisic acid. Plant Physiol. 2004;134:1439-49. https://doi.org/10.1104/pp.103.037614.

Sato H, Takasaki H, Takahashi F, Suzuki T, luchi S, Mitsuda N, et al. Arabidopsis thaliana NGATHA1 transcription factor induces ABA biosynthesis by activating NCED3 gene during dehydration stress. Proc Natl Acad Sci U S A. 2018;115:E11178-87. https://doi.org/10.1073/pnas.1811491115.

Tan L, Liu Q, Song Y, Zhou G, Luan L, Weng Q, et al. Differential function of endogenous and exogenous abscisic acid during bacterial patterninduced production of reactive oxygen species in Arabidopsis. Int J Mol Sci. 2019. https://doi.org/10.3390/ijms20102544.

Thatcher LF, Gardiner DM, Kazan K, Manners JM. A highly conserved effector in Fusarium oxysporum is required for full virulence on Arabidopsis. Mol Plant Microbe Interact. 2012;25:180-90. https://doi.org/10.1094/ mpmi-08-11-0212.

Tsugama D, Liu S, Takano T. A bZIP protein, VIP1, is a regulator of osmosensory signaling in Arabidopsis. Plant Physiol. 2012;159:144-55. https://doi.org/ 10.1104/pp.112.197020.

Umezawa T, Sugiyama N, Mizoguchi M, Hayashi S, Myouga F, YamaguchiShinozaki K, et al. Type $2 \mathrm{C}$ protein phosphatases directly regulate abscisic acid-activated protein kinases in Arabidopsis. Proc Natl Acad Sci U S A. 2009;106:17588-93. https://doi.org/10.1073/pnas.0907095106.

Wang W, Liu N, Gao C, Rui L, Tang D. The Pseudomonas Syringae effector AvrPtoB associates with and ubiquitinates Arabidopsis exocyst subunit EXO70B1. Front Plant Sci. 2019;10:1027. https://doi.org/10.3389/fpls.2019. 01027.

Washington EJ, Mukhtar MS, Finkel OM, Wan L, Banfield MJ, Kieber JJ, et al. Pseudomonas syringae type III effector HopAF1 suppresses plant immunity by targeting methionine recycling to block ethylene induction. Proc Natl Acad Sci U S A. 2016;113:E3577-86. https://doi.org/10.1073/pnas. 1606322113.

Xiao F, He P, Abramovitch RB, Dawson JE, Nicholson LK, Sheen J, et al. The $\mathrm{N}$-terminal region of Pseudomonas type III effector AvrPtoB elicits Ptodependent immunity and has two distinct virulence determinants. Plant J. 2007;52:595-614. https://doi.org/10.1111/j.1365-313x.2007.03259.x.

Xiao X, Cheng X, Yin K, Li H, Qiu JL. Abscisic acid negatively regulates postpenetration resistance of Arabidopsis to the biotrophic powdery mildew fungus. Sci China Life Sci. 2017;60:891-901. https://doi.org/10.1007/ s11427-017-9036-2.

Xin XF, He SY. Pseudomonas syringae pv. tomato DC3000: a model pathogen for probing disease susceptibility and hormone signaling in plants. Annu Rev Phytopathol. 2013;51:473-98. https://doi.org/10.1146/annur ev-phyto-082712-102321.
Xin XF, Nomura K, Aung K, Velásquez AC, Yao J, Boutrot F, et al. Bacteria establish an aqueous living space in plants crucial for virulence. Nature. 2016;539:524-9. https://doi.org/10.1038/nature20166.

Xu N, Luo X, Wu W, Xing Y, Liang Y, Liu Y, et al. A plant lectin receptor-like kinase phosphorylates the bacterial effector AvrPtoB to dampen its virulence in Arabidopsis. Mol Plant. 2020;13:1499-512. https://doi.org/10.1016/j.molp. 2020.09.016.

Yang B, Wang Y, Guo B, Jing M, Zhou H, Li Y, et al. The Phytophthora sojae RXLR effector Avh238 destabilizes soybean Type2 GmACSs to suppress ethylene biosynthesis and promote infection. New Phytol. 2019;222:425-37. https://doi.org/10.1111/nph.15581.

Zhang Z, Chen H, Huang X, Xia R, Zhao Q, Lai J, et al. BSCTV C2 attenuates the degradation of SAMDC1 to suppress DNA methylation-mediated gene silencing in Arabidopsis. Plant Cell. 2011;23:273-88. https://doi.org/10. 1105/tpc.110.081695.

Zipfel C. Plant pattern-recognition receptors. Trends Immunol. 2014;35:345-51. https://doi.org/10.1016/j.it.2014.05.004.

\section{Publisher's Note}

Springer Nature remains neutral with regard to jurisdictional claims in published maps and institutional affiliations.
Ready to submit your research? Choose BMC and benefit from:

- fast, convenient online submission

- thorough peer review by experienced researchers in your field

- rapid publication on acceptance

- support for research data, including large and complex data types

- gold Open Access which fosters wider collaboration and increased citations

- maximum visibility for your research: over 100M website views per year

At BMC, research is always in progress.

Learn more biomedcentral.com/submissions 